\title{
Feiz ha Breiz (1865-1884) ou la genèse d'une langue journalistique
}

Feiz ha Breiz (1865-1884) or the genesis of a journalistic language

\section{Cédric Choplin}

\section{Q OpenEdition}

\section{Journals}

Édition électronique

URL : https://journals.openedition.org//bl/2112

DOl : $10.4000 / \mathrm{lbl} .2112$

ISSN : 2727-9383

Éditeur

Université de Bretagne Occidentale - UBO

\section{Édition imprimée}

Date de publication : 1 novembre 2011

Pagination : 19-35

ISBN : 978-2-901737-92-6

ISSN : 1270-2412

\section{Référence électronique}

Cédric Choplin, «Feiz ha Breiz (1865-1884) ou la genèse d'une langue journalistique », La Bretagne Linguistique [En ligne], 16 | 2011, mis en ligne le 01 mai 2021, consulté le 22 mai 2021. URL : http:// journals.openedition.org//bl/2112 ; DOl : https://doi.org/10.4000/lbl.2112

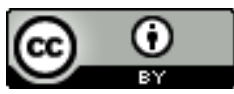

La Bretagne Linguistique est mise à disposition selon les termes de la Licence Creative Commons Attribution 4.0 International. 


\title{
Feiz ha Breiz (1865-1884) ou la genèse d'une langue journalistique
}

\begin{abstract}
$\boldsymbol{H}_{\text {eiz }}$ blié entre 1865 et 1884 . Il participe, de toute évidence, au mouvement des Semaines Religieuses, ces hebdomadaires catholiques assez bon marché qui, profitant de l'assouplissement des lois et règlements sur les journaux, fleurissaient dans chaque évêché à la fin du Second Empire. Feiz ha Breiz se distingue pourtant de ces journaux sur deux points : premièrement, et contrairement aux autres Semaines Religieuses rédigées presque exclusivement en français, Feiz ha Breiz est écrit en breton; deuxièmement, Feiz ha Breiz ne se contente pas de traiter de la vie religieuse dans son diocèse. En effet, il s'agit de donner aux lecteurs bretonnants des nouvelles de tous les pays et de les instruire sur tout comme l'indique le sous-titre de la publication (Kelou a bep bro ha kenteliou var pep tra, digasset bep sul da guement Christen a goms ar brezounek).

Cette ambition d'informer et d'instruire les Bas-Bretons avait surtout une visée apologétique et politique puisqu'il s'agissait avant tout de défendre l'Église et les idées monarchistes et de détourner les catholiques des «idées impies et nouvelles» véhiculées par la presse en langue française, elle aussi en pleine expansion dans le Finistère.
\end{abstract}

* Docteur en celtique, CRBC Rennes $2 /$ ueb 
L'idée de lancer la publication d'un hebdomadaire en breton pour défendre l'Église et le légitimisme s'était pour ainsi dire imposée d'elle-même à Léopold de Léséleuc, vicaire général, et à son évêque, Mr Sergent. En effet, il existait déjà une publication en français, l'Impartial du Finistère, qui défendait les mêmes idées et dont le propriétaire imprimeur, Arsène de Kerangal, se vit confier l'administration et l'impression de Feiz ha Breiz. De plus, comme l'ont montré les travaux de Fañch Broudic ${ }^{1}$, le breton était à l'époque la langue de communication ordinaire de l'immense majorité de la population.

\section{De la difficulté de créer un journal en breton en 1865}

\section{"Brezhoneg beleg» contre réforme de Le Gonidec}

Restait donc à définir dans quel breton serait rédigée la nouvelle publication. De façon à peine schématique, on pouvait alors discerner deux camps chez ceux qui se piquaient d'écrire en breton : les tenants de ce que l'on appelle le «brezhoneg beleg», breton de prêtre selon l'expression de Camille Le Mercier d'Erm, un galimatias francobreton, semblaient irréductiblement opposés aux tenants de la réforme initiée par le Gonidec au début du XIX ${ }^{\mathrm{e}}$ siècle. Pour aller vite, la réforme de Le Gonidec consistait à épurer le breton des emprunts trop visibles au français et à les remplacer soit par des mots plus anciens, plus «celtiques» mais sortis de l'usage quotidien, soit par des néologismes formés à partir de racines anciennes ou du gallois.

La question était d'autant plus sensible que la version bretonne des Annales de la Propagation de la Foi en avait déjà fait les frais. Quelque vingt ans plus tôt, en 1843, Monseigneur Graveran, évêque de Quimper et bretonnant accompli, convaincu de la réforme de Le Gonidec, avait confié à l'abbé Jean-Guillaume Henry, aumônier à l'hôpital de Quimperlé, la rédaction de la revue. Une bonne partie du clergé manifesta ouvertement son mécontentement face à ces nouveautés linguistiques. L'abbé Henry dut certes en rabattre un peu mais cela ne l'empêcha pas d'être évincé peu après la disparition de son

1. Fañch BROUDIC, La pratique du breton de l'Ancien-Régime à nos jours, PUR, 1995. 
protecteur en 1855. Il est alors remplacé par le chanoine Alexandre, peu soupçonnable de purisme comme le montre cet exemple cité par Lukian Raoul : «Ordrenanç. Desir consail Breuriez ar Feiz... ${ }^{2}$ »

Dans cette première bataille, les tenants du «brezhoneg beleg» l'avaient donc emporté et si le tirage de Lizirou Breuriez ar Feiz augmenta, sa qualité littéraire déclina en proportion.

\section{Le bretonisme}

Vingt ans plus tard, un changement de génération a eu lieu dans le clergé paroissial et la sympathie de la jeune génération, enflammée par la lecture du Barzaz Breiz, va au bretonisme. Nous retiendrons ici, comme définition du bretonisme, celle qu'en donne Michel Lagrée :

«Sous ce terme générique, je retiendrai ici l'ensemble des attitudes qui vont au-delà de l'utilisation de la langue comme simple outil de communication et confère à cette utilisation une signification idéologique avec la dimension d'un engagement. À l'usage simplement véhiculaire se superpose en effet une volonté commune d'apologie et de défense de la langue bretonne, dont l'intérêt pour mon propos tient aux liens, souvent étroits avec l'apologie et la défense de la foi catholique ${ }^{3}$.»

Kersalé, qui signe parfois du pseudonyme An Ermit, pose dans le numéro du 8 mai 1869 la question : "Ha mad eo d'eomp-ni delc'her d'or brezouneg ${ }^{4}$ ?» Dans sa réponse, il développe ses arguments en faveur du breton et récite son credo bretoniste. Constatant l'affaiblissement de la foi en France d'une part et la vigueur de celle-ci en Bretagne d'autre part, il conclut que c'est la langue qui sert de rempart face aux idées nouvelles et impies. Un peu plus loin, il expose que la langue bretonne trouve son origine dans la destruction de Babel : l'origine de la langue bretonne est donc divine. A contrario, le français dérive du latin qui, comme le breton, est supposé d'origine babélienne.

2. Lizeroù Breuriez ar Feiz n ${ }^{\circ}$ 57, novembre 1853. Cité par Lukian RAOUL, Un siècle de journalisme breton, Le Guilvinec, Le Signor, 1981, p. 84.

3. Michel Lagrée, Religion et cultures en Bretagne, Paris, Fayard, 1992, p. 233.

4. «Est-il bon pour nous de conserver notre breton?» 
Ar brezoneg a zo greet gan Doue; eun tam dishevelebet eo, evid gwir, gan an dud, mes roudou dorn Doue evel-kent a jom anat enn-ha c'hoaz; e lec'h ar galleg a zo greet gan an dud, voar eur font, evid gwir, deuet digan Doue, mes ar font-ze a zo re guzet hiryo evid beza remerket gan ar gomun.

(F\&B n 223 08/05/1869)

(Le breton a été créé par Dieu. Il a été quelque peu déformé, par les hommes, c'est vrai; mais la trace de la main de Dieu reste tout de même encore évidente en lui. Contrairement au français qui a été créé par l'homme sur une base divine, il est vrai; mais cette base est aujourd'hui beaucoup trop cachée pour être remarquée par le commun des mortels.)

De ce fait, la corruption du breton par des mots français tient du blasphème et Kersalé ne se prive pas pour le dire :

Respetit ivez ho prezoneg, na yit ket da gemmeska gan-t-ha kemment a c'heryou galleg, evel ma ra lod, evel ma ra eur braz euz ar re ac'hannoc'h hag a voar eun tam galleg benag : mall eo gan-t-ho, mar plij, diskouez ec'h ouzont eur gerig galleg benâg, hag e fourront anezho muia ma c'hellont en ho brezoneg. An draze, va mignoned, a zo sklabeza, saotri yez kaer on Tadou.

(Respectez aussi votre langue bretonne, n'allez pas la mélanger avec tant de mots français, comme le font certains, comme le fait la majorité d'entre vous qui savez un peu de français. Ils ont hâte, s'il vous plaît, de montrer qu'ils savent quelques mots et les fourrent tant qu'ils peuvent dans leur breton. Ceci, mes amis, c'est souiller, salir la belle langue de nos ancêtres.)

La cause bretoniste semblait donc entendue mais il ne faut pas se méprendre. En effet, bien que Kersalé ait été l'un des rédacteurs les plus fidèles du journal, il n'en était pas le rédacteur en chef.

\section{Goulven Morvan : un bretoniste pragmatique}

La rédaction du journal avait été confiée dès ses débuts à Goulven Morvan. Ce fils d'agriculteur analphabète de la Forêt-Landerneau dans le Léon était né en 1819. Ce n'est qu'à l'âge de 32 ans, en 1851, qu'il fut ordonné prêtre. Son initiation au bretonisme se fit au petit séminaire où, en sus d'un solide cursus en humanités, il suivit le cours d'Auguste Lamarque. Ce cours, d'après les recherches menées 
par Ronan Calvez ${ }^{5}$, avait le Barzaz Breiz comme support principal. C'est probablement dans ce cours qu'il rencontra Gabriel Morvan, son condisciple de dix ans son cadet et qui plus tard le seconda avant de le remplacer à la tête de Feiz ha Breiz.

C'est là aussi qu'il rencontra Léopold de Léséleuc de Kerouara qui y était son professeur et qui le remarqua au point de s'en souvenir quand il recherchait un rédacteur en chef pour Feiz ha Breiz. Il avait toutes les qualités requises. Excellent bretonnant, travailleur, pragmatique et affable; il saurait se faire apprécier de tous. Léopold de Léséleuc n'a pas dû être déçu.

Dans le premier éditorial de la sixième année de l'hebdomadaire ( $F \& B n^{\circ} 262$ 05/02/1870), Goulven Morvan après s'être félicité du succès de sa publication, expose non sans humour les dilemmes linguistiques auxquels il est confronté.

D'un côté, les tenants de l'orthographe de Le Gonidec le critiquent sévèrement :

Ervez al liziri a resevomp a bep tu, e plij d'an darnvuia euz a lennerien ar gazeten, ken ar brezoneg a zo enhi, ken an traou a laker e brezonek. Mez etouez calz e caver ato hiniennou hag a zo diez ober ho lavar outho. Bez'ez euz unan bennag hag en em glem dre na laker ket er gazeten ar brezonek coz, ar geriou-ze eat da fall, da lavaret eo, ne dint mui comzet e corn ebet euz a Vreiz; brezonek coz hag a zavas cri ha clem en he enep pa en em lakejot da scrifa lizerou Breuriez ar Feiz er brezonek-ze, en hevelep doare ma voue red he lezel a gostez, evit scrifa brezoneg evel a gomzer er vro. Ar re-ma en em glem c'hoaz dre ne scrifer ket tre ervez reiz pe giz scrifa an aotrou ar Gonidec.

(D'après les lettres que nous recevons de toutes parts, le journal plaît à la majorité de ses lecteurs, tant par son breton que par les choses que l'on y raconte en breton. Mais parmi un grand nombre on en trouve toujours quelques-uns qu'il est difficile de contenter. Il y en a qui se plaignent que l'on n'y mette pas de vieux breton, ces mots tombés en désuétude, c'est-à-dire qui ne sont plus employés nulle part en Bretagne. Ce vieux breton contre lequel s'élevèrent cris et plaintes quand on se mit à rédiger Lizerou Breuriez ar Feiz dans ce breton. C'est ainsi que l'on

5. Ronan CALVEZ, «Un paysanisme breton » Feiz ha Breiz et la société bretonne (1865-1875), Brest, Mémoire de maîtrise, 1993. 
dut l'abandonner pour écrire le breton comme il se parle dans le pays. Les mêmes se plaignent parce que l'on n'écrit pas tout à fait selon l'orthographe de M. le Gonidec.

D'un autre côté les tenants du «brezhoneg beleg» ne sont pas non plus avares de reproches :

Bez'ez euz hiniennou all hag a garfe e ve laket er gazeten, brezoneg evel a gaver en eul levr bennak moullet brema ez euz antercant pe driugent vloas, da lavaret eo, eur iez hag a zo an anter euz ar geriou enha gallek; ha ne ket ar geriou epken, mes c'hoaz an dro a roet d'ar c'homzou, en hevelep doare ma ve red gouzout ar galleg evit gouzout petra a leveront. C'hoaz e falfe dezho e ve scrifet ar brezonek gant ar pez a c'halvont ortograf latin.

(Il y en a d'autres qui aimeraient que l'on mette dans le journal du breton comme on peut le trouver dans quelques livres imprimés il y a 50 ou 60 ans, c'est-à-dire dans une langue qui contient pour moitié des mots français. Et il ne s'agit pas que des mots puisque la syntaxe est telle qu'il est nécessaire de connaître le français pour pouvoir comprendre ce qu'ils disent. De plus il faudrait que nous écrivions le breton selon ce qu'ils appellent l'orthographe latine.)

Si Goulven Morvan ne prend même pas la peine de répondre aux tenants $\mathrm{du}$ «brezhoneg beleg», estimant que cette réponse était contenue dans sa présentation de leurs arguments; il répond de manière longue et nuancée aux tenants de Le Gonidec.

Tout d'abord, il les invite à constater que la différence entre l'orthographe de Le Gonidec et la sienne est mince. Il justifie ces différences par le fait que son lectorat est majoritairement constitué de ruraux, peu habitués à des lettres comme le W. Il précise aussi qu'il ne parle ici qu'en son nom puisque les collaborateurs réguliers ou occasionnels sont laissés libres d'utiliser l'orthographe qu'ils préfèrent. Ensuite, il leur explique sagement que bien qu'il apprécie les anciens mots qu'affectionnent tant les puristes, il n'entend pas faire la même erreur que Lizerou Breuriez ar Feiz à ses débuts.

Me garfe ervad e teufe adarre da vad calz a gomzou a zo eat da fall, hag a zo digaset geriou galleg en ho leac'h. Me a zigas ive en dro meur a c'her evelse pa gaf din e cavan tro d'ho lacat 
da dremen. Hogen kementse a deufe kentoc'h da benn dre al levriou. An den divar ar meaz, o lenn eul levr, n'her c'hompreno ket marteze ar vech kenta, mes al levr a jom etre he zaouarn, hag e compreno an eil pe an drede... pe an decved guech. Ne ket evelse evit eur gazeten; houma a rank beza comprenet ar vech kenta, ha zoken ep poan, anez e teufet buan da enoui oc'h he lenn. Comz a ran ama dreist oll euz ar goueriadet, euz an dud divar ar meaz. Ar re-ma n'int ket boas da studia, da bleustri evit gouzout a beleac'h e teu eur ger ha ne anavezont ket. Pa gavont eta geriou evelse, mar deo red dezho chom da zonjal ha da studia, setu evitho eun dra poaniusoc'h eget al labourou a reont en ho farkeier. Ar pennad lenn-ze a dle beza evitho eur blijadur, eun tremen amzer; rak Feiz-ha-Breiz a zo bet savet dreist pep tra evit ho c'helen, evit deski dezho aleiz a draou ha ne ouzont ket, hag a rafe gouscoude vad dezho da c'houzout.

(J'aimerais beaucoup que soient réhabilités un grand nombre de ces mots oubliés qui sont remplacés par des mots français. J'en apporte moi-même quelques-uns quand je trouve l'occasion de les faire passer. Or, ceci se fera surtout grâce aux livres. Le campagnard, qui lit un livre, ne le comprendra peut-être pas du premier coup mais le livre reste entre ses mains et il le comprendra à la seconde, à la troisième... ou à la dixième fois. Il n'en va pas de même pour le journal car celui-ci doit être compris du premier coup et sans peine. Sinon, on s'ennuierait vite à le lire. Je parle surtout ici des paysans. Ceux-ci ne sont pas habitués à étudier, à chercher à savoir d'où vient un mot qu'ils ne connaissent pas. Quand ils trouvent de tels mots et s'ils doivent réfléchir ou étudier, voilà pour eux une tâche plus pénible que le travail qu'ils font dans leurs champs. Cet article doit être pour eux un plaisir, un passe-temps car Feiz ha Breiz a été créé avant tout pour les instruire, pour leur apprendre beaucoup de choses qu'ils ne savent pas et qu'il leur serait profitable pourtant de savoir.)

Goulven Morvan se positionne donc pour un purisme modéré. Son objectif en matière de langue est donc d'offrir à son lectorat peu instruit des textes de bonne qualité mais faciles à lire.

Nous avons vu que pendant les dix années durant lesquelles Goulven Morvan a été à la tête de Feiz ha Breiz, chacun des collaborateurs pouvait utiliser l'orthographe qui lui plaisait. Cette tolérance ouvrait au débat un espace que Kersalé ne manqua pas d'occuper en juin 1867. Bien qu'il expose les principes de sa «meilleure ortho- 
graphe» pour le breton sous forme dialoguée et vivante tant que faire se peut, Feiz ha Breiz (Goulven Morvan ou Kersalé lui-même?) se fend d'une remarque en italique placée sous le titre de l'article :

Ar pennad-ma na zell nemed ouz an dud desket pe gouizieg d.l.e. nemed ouz ar re a voar, ouspenn ar brezoneg, ar galleg ha yezou all.

(Cet article ne s'adresse qu'aux gens instruits ou savants, c'est-à-dire seulement à ceux qui savent le français et d'autres langues en plus du breton.)

Dans ces articles des 22 et 29 juin, il explique en comparant avec les autres langues celtiques que «gant» doit s'écrire sans T, qu'il faut supprimer le $\mathrm{P}$ de la conjugaison à la première personne du pluriel, etc.

Dans le numéro du 27 août, ces deux articles reçoivent d'un La Villemarqué au faîte de sa gloire une réponse élégante mais cinglante.

Va Aotrou mad ha va mignon,

Ann tabud etre Iann ha Per, hoc'h euz lekeat ker brao dre skrid, am euz lennet gant plijadur, evit-han da zellet hebken, eme-c'houi, ouz an dud desket pe gouizyeg.

(Mon cher bon monsieur et ami,

J'ai lu avec grand plaisir le débat entre Yann et Pierre que vous avez si joliment écrit bien qu'il ne s'adresse, à ce que vous dites, qu'aux gens instruits ou savants.)

Après avoir écrit qu'il aurait préféré lire de sa plume une belle chanson dont il a le secret (à chacun son domaine), il passe à l'offensive dans une longue lettre où perce une modestie feinte.

Nemed eleac'h rei ma mennoz-me, hag a zo netra, am euz losket ar gomz gant ar re ho deuz va c'helennet, ha setu ar pez a lavar ann doktored-ze, abaoe hon amzer-ni beteg ann amzeriou pella, diwarbenn GANT ha diwarbenn OMP.

(Mais au lieu de vous donner mon opinion personnelle, qui ne vaut rien, j'ai laissé la parole à ceux qui m'ont instruit. Et voici ce que disent ces savants depuis notre temps jusqu'aux temps les plus reculés au sujet de GANT et de OMP.) 
C'est ainsi que sur deux colonnes entières, il ensevelit le malheureux dialogue entre Yann et Per sous des références de poids. Il est évident que si Kersalé a pu se sentir flatté de recevoir une réponse d'un homme aussi illustre que La Villemarqué, il a dû déchanter en la lisant. Quoi qu'il en soit, son article suivant (17 septembre 1867) sur l'orthographe du breton dans lequel il expose les sons que font les lettres $\mathrm{G}, \mathrm{S}$ et $\mathrm{W}$ dans ses écrits tient en à peine dix lignes. Le 28 décembre 1867, Feiz ha Breiz publie une lettre de Kersalé à La Villemarqué. On y apprend que Feiz ha Breiz a refusé de publier une précédente lettre et estime ne pas avoir été créé pour abriter ce genre de débats savants. Kersalé pose donc une bonne dizaine de questions sur le breton à La Villemarqué et l'invite à lui répondre dans l'Impartial $d u$ Finistère. Ces questions portent essentiellement sur l'origine du breton et son orthographe. Le ton est nettement polémique puisque le registre employé est celui du gouren.

Même si la liberté reste de mise chez les collaborateurs du journal, on constate qu'au fil des ans s'élabore une « orthographe Feiz ha Breiz» plus ou moins respectée par tous. Dans deux articles (19 et 26 février 1876) le chanoine Gabriel Morvan, qui avec Nedélec a pris la suite de Goulven Morvan, explique que les collaborateurs du journal devront désormais se conformer à l'orthographe de Le Gonidec à quelques exceptions près dont il dresse la liste. En fait, il simplifie l'orthographe de Le Gonidec en lui ôtant les signes diacritiques incompréhensibles, corrige quelques incohérences et la «déléonise» quelque peu eu égard à ses lecteurs cornouaillais.

Quand Gabriel Milin, écrivain bien connu du public bretonnant, reprend le journal en 1883, il impose à tous ceux qui lui envoient des papiers d'écrire comme lui ou d'accepter de voir leurs écrits corrigés. Selon lui, il doit en être ainsi pour que le breton de ce journal soit uni comme sa foi.

L'orthographe est une chose, le style en est une autre.

\section{Le style de Feiz ha Breiz}

On peut s'étonner de prime abord d'entendre parler du style de Feiz ha Breiz comme s'il s'agissait là de quelque chose d'immuable malgré la succession des rédacteurs et des collaborateurs pendant dix-neuf ans. Il est vrai que certains articles sautent aux yeux du 
lecteur en raison de traits dialectaux évidents ou d'un style d'écriture particulier. Mais s'ils sont si faciles à repérer, c'est bien parce que Feiz ha Breiz montre une unité de langue et de style. La raison en est assez simple. Premièrement, c'est le même homme (Goulven Morvan) qui a été à la tête du journal pendant les dix premières années, c'est-à-dire pendant plus de la moitié de son existence. De plus, il apparaît au détour de plusieurs articles que Goulven Morvan devait bien souvent remplir le journal tout seul ou avec une équipe de collaborateurs réguliers fort réduite. Le style de Feiz ha Breiz est donc avant tout le style de Goulven Morvan.

\section{La suprématie du léonais}

En introduction à leur recueil de textes choisis dans Feiz ha Breiz ${ }^{6}$, Jean Le Dû et Yves Le Berre se sont livrés à une étude des traits dialectaux du breton de Goulven Morvan. Pour ce faire, ils ont constitué une liste de mots courants pouvant présenter des variations lexicales ou phonétiques présents dans les articles de Goulven Morvan et dans l'Atlas Linguistique de Basse-Bretagne de Pierre Le Roux. En traçant les isoglosses, il est apparu que la langue de Feiz ha Breiz est caractéristique du breton parlé dans un quadrilatère Landeda (point 3 de l'ALBB), Bohars (point 9), Trémaouézan (point 10) et Saint-Frégant. Or, La Forest-Landerneau est inscrite dans cet espace.

L'influence léonarde sur le breton de Feiz ha Breiz était d'autant plus forte que c'est sur ce breton que Le Gonidec avait basé ses travaux, que même un Cornouaillais comme La Villemarqué estimait ce parler plus pur et élégant.

Cependant, bien qu'étant léonard comme une bonne partie du clergé et des collaborateurs de Feiz ha Breiz, Goulven Morvan était installé dans la région de Quimper. Il était donc bien placé pour savoir quels traits de son breton pourraient être mal ou pas compris de son lectorat cornouaillais.

On peut donc conclure ici avec Jean Le Dû et Yves Le Berre que «c'est donc véritablement de sa langue maternelle que le directeur de Feiz ha Breiz a fait cet instrument de communication qu'il a poli au

6. Jean Le Dû, Yves Le Berre, Textes choisis dans Feiz ha Breiz, Studi n 11, Brest, avril 1979. 
cours des dix années pendant lesquelles il a été le principal rédacteur de cette revue. D'un parler authentique il a su faire une langue écrite susceptible d'être comprise dans une région très étendue, l'évêché de Quimper et de Léon».

La stabilité de la langue de Feiz ha Breiz s'explique aussi par le fait que tous les rédacteurs en chef qui ont succédé à Goulven Morvan étaient originaires du Léon ainsi que $46 \%$ des collaborateurs attestés pour la période 1865-1875.

\section{Décrire l'ailleurs}

Décrire des objets ou expliquer des concepts étrangers de manière aisément compréhensible n'est jamais chose aisée. Elle l'est d'autant moins quand on doit le faire à des gens peu instruits et dans une langue habituellement et depuis longtemps dédaignée des intellectuels. Or, ce sont ces derniers qui en général introduisent de nouveaux mots-concepts ou donnent à des mots usuels un nouveau sens dans leur domaine d'activité. Le breton, en l'occurrence n'avait que peu bénéficié de cet apport. On peut certes poser là un bémol en ce qui concerne le vocabulaire religieux mais le «brezhoneg beleg» était-il vraiment du breton en tant que langue de communication (an examin a gonscience...). La tâche des rédacteurs de Feiz ha Breiz s'avérait donc ardue.

Comment donc traduire dans une langue des concepts et des choses qui sont étrangers à son environnement habituel? La première solution, la plus simple est évidemment l'emprunt qui n'est possible que si les deux langues ont un système phonologique assez proche ou au prix de modifications importantes de la prononciation. De plus, les emprunts se font souvent de façon indirecte, c'est-à-dire qu'ils passent par une tierce langue comme le français dans le cas de Feiz ha Breiz. Les rédacteurs ne citant que rarement leurs sources, il est bien souvent impossible de savoir si un article est une traduction ou un texte original en breton à l'instar de cette lettre envoyée par un missionnaire breton en Kabylie :

Ouspen e caver eur benvec, hanvet Tobokolt, hag a zo henvel braz euz ar benvec na hanver ket e Breiz-Izel, hag a gaver peurvuia dindan ar gueleou. Ann Tobokolt a zo eur pot-pri eta; meur a vicher e renk ober; en em zervicha a reer outhan evit ober meur a dra. 
(On trouve un autre ustensile, appelé Tobokolt, qui ressemble énormément à un objet qu'on ne nomme pas en Bretagne et qu'on trouve le plus souvent sous les lits. Le Tobokolt est donc un pot de terre qui doit avoir plusieurs utilisations ; on s'en sert à divers usages ${ }^{7}$.)

L'autre technique pour nommer des objets exotiques est de recourir au néologisme et les rédacteurs de Feiz ha Breiz sont passés maîtres dans l'art de créer de nouveaux mots à partir de mots bien connus et courts. Ces néologismes, les plus faciles à saisir, comme «liamfrouez» (lien-fruit) pour liane ne sont généralement pas traduits en français (à quoi bon puisque le lecteur n'est pas supposé le savoir) et seul le contexte en explicite le sens :

Gant eun taol bouc'hal e troc'haz eur scoden euz an doare liamfrouez a gaver er c'hoajou-ze, hag a zo ker stang ma reont eun doare rouejou, ha ne deus nemet ar c'houezidi hag a c'helfe en em denna dreizho. Al liamfrouez-ze a zo ker guen ha ker soubl hag eur gorden, hag ober a rer gantho eno kement a rer gant kerdin er broiou seven.

(D'un coup de hache il coupa un bout de cette sorte de lianes que l'on trouve dans ces bois et qui sont si denses qu'elles forment comme des filets et que seuls les sauvages pourraient traverser. Ces lianes sont aussi blanches et souples qu'une corde et on les utilise tout à fait comme les cordes dans les pays civilisés ${ }^{8}$.)

Parfois, cependant, d'autres objets exotiques sont traduits et expliqués longuement, surtout si cette explication a aussi un objectif qui dépasse la simple définition :

Ar c'hristen-ze, hanvet Yank, a voa bet barnet da zouguen evit bepret ar skeul-c'houzoug (la cangue). Ar re a lenn liziri Breuriez ar Feiz a voar petra eo ar skeul-c'houzoug. Bez'ez eo daou dam coat pe daou bost stag an eil oc'h eguile gant diou vaz evel eur c'hravaz pe eur brancardou. Etre an diou vaz e tremener ar penn, hag an daou bost a zisken var an diou scoas. Avechou eo henvel oc'h eur golo taol a ve graet eun toull er c'heiz anezhan evit tremen ar penn. Mar sonjer penaus ar skeul-c'houzoug a

7. $\mathrm{F} \& \mathrm{~B} \mathrm{n}^{\circ} 23$ (7 juin 1879$)$.

8. F\&B n ${ }^{\circ} 458$ (8 novembre 1873 ). 
bouez avechou bete pevar ugent, bete kant lur, penaus an hini so laket ar skeul-ze dezhan ne all ket sevel he zaouarn d'he c'hinou, ne all ket sellet oc'h an douar, ne all ket gourvez...e c'heller gouzout eun dra bennag pebez merzerinti eo dougen ar skeulc'houzoug-ze.

(Ce chrétien, appelé Yank, avait été condamné à porter à tout jamais l'échelle de cou (la cangue). Ceux qui lisent Lizerou Breuriez ar Feiz savent ce qu'est une échelle de cou. Elle consiste en deux morceaux de bois ou deux poteaux attachés l'un à l'autre par deux bâtons comme une civière ou des brancards. On passe la tête entre ses deux bâtons et les deux poteaux descendent sur les épaules. Elle ressemble parfois au plateau d'une table dans le centre duquel on a fait un trou pour passer la tête. Quand on sait que l'échelle de cou pèse parfois entre 80 et jusqu'à 100 livres, que celui qui doit la porter ne peut ni lever les mains à sa bouche, ni regarder par terre, ni s'allonger... On peut imaginer le martyre que constitue le port de cette échelle de $\mathrm{cou}^{9}$.)

Notons que par la suite, Feiz ha Breiz utilisera le mot «Kang, ar c'hang» passé en breton par l'intermédiaire du français concurremment avec le néologisme skeul-c'houzoug / échelle de cou maintenant compris de tous ${ }^{10}$.

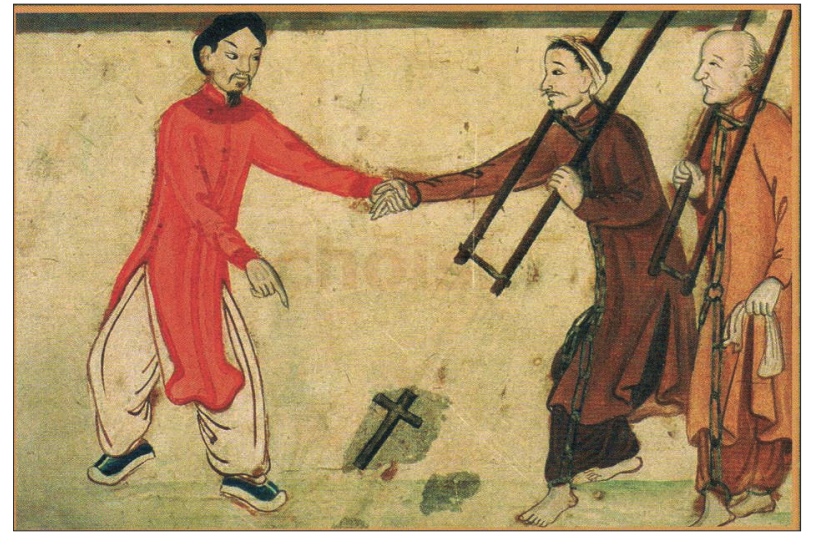

9. $\mathrm{F} \& \mathrm{~B} \mathrm{n}{ }^{\circ} 90$ (20 octobre 1866$)$.

10. Illustration : Martyre de Paul Khoan, Pierre Hiêu et Jean-Baptiste Thành (détail). Les prisonniers comparaissent devant le préfet et refusent de fouler la croix, 1837, anonyme vietnamien. Papier marouflé sur toile, collection Missions Étrangères de Paris. Avec leur aimable autorisation. 
L'un des grands mérites de Feiz ha Breiz, qui entendait traiter de tout en breton est d'avoir donné aux écrivains, lexicologues et grammairiens bretons des méthodes et une base sur lesquelles ils peuvent s'appuyer encore aujourd'hui pour permettre au breton si ancien de rester un outil de communication performant dans un monde qui change si vite. Cette méthode consiste à vrai dire en peu de choses : pour qu'un néologisme soit acceptable, il doit être court (deux ou trois syllabes) et forgé à partir de mots connus. Autant «rozelloù-glav» pour essuie-glaces aurait très bien pu se trouver sous la plume de Goulven Morvan, autant «fiñskeudenndi» pour cinéma l'aurait très certainement laissé dubitatif. Il est important de noter que Goulven Morvan et ses disciples adaptent plus qu'ils ne traduisent. C'est la raison pour laquelle, lorsqu'ils ne trouvent pas en breton de mot compréhensible équivalent à celui du texte original en français, ils utilisent une périphrase très pédagogique. Ainsi, les nomades sont des gens «qui vont d'un lieu à l'autre sans rester très longtemps quelque part ${ }^{11} \gg$. Cette moindre utilisation des substantifs concoure évidemment à la vigueur du style de Feiz ha Breiz.

\section{Un style alerte}

L'objectif des rédacteurs de Feiz ha Breiz est d'instruire les catholiques bas-bretons en les distrayant. La forme est donc très importante. Elle est d'autant plus importante que Feiz ha Breiz n'est pas illustré alors que des revues catholiques comme L'Ouvrier regorgent de gravures qui happent le lecteur.

À l'exception de l'éditorial, les articles sont donc le plus souvent présentés sous des formes qui plaisent au lectorat rural : le conte, la dispute ou la poésie chantée ou non. La conclusion est toujours très morale et simple. Le Breton a raison de tenir à sa langue, à ses traditions, à sa religion, à ses prêtres et à son roi (Henri V); l'ouvrier, le bourgeois voltairien, les protestants, les républicains et les savants athées sont fustigés ou tournés en ridicule.

La forme dialoguée est très souvent privilégiée et nous en avons vu un exemple avec Kersalé et la question de l'orthographe. Ainsi la rencontre entre l'évêque d'Alger et un marabout dans un hôpital de

11. Exemple cité par Jean LE Dû, Yves Le BerRe, Textes choisis dans Feiz ha Breiz, op. cit. 
campagne après une bataille permet à Feiz ha Breiz de donner sous une forme exotique et vivante un rappel du cours de catéchisme sur le dogme trinitaire. À la fin de ce dialogue, le marabout embrasse l'évêque avec effusion et le remercie de l'avoir éclairé ${ }^{12}$.

Après le départ de Goulven Morvan en 1875, Feiz ha Breiz prend un tour de plus en plus politique. Son contenu s'en trouve évidemment modifié avec un ton de plus en plus polémique. La virulence atteint parfois des sommets proprement inouïs les lendemains de défaites électorales. Dans le numéro 34 du 20 octobre 1877, TorrHe-Benn félicite les Léonards et les électeurs de l'arrondissement de Quimperlé qui ont «bien voté». Il se tourne ensuite vers les autres et déclare :

D'ar rest euz an Departamant ne velomp ket guelloc'h coumplimant da ober eget an hini a rea guechall sant Stephan d'ar Juzevien.

Pennou calet, discouarn potin, calounou dir ma'z oc'h, emezhan, c'hui zo bet enebourien hed ar veach da spered Doue; an Den Just oc'h euz lakeat d'ar maro ; he lezen, douget beteg ennoc'h var diouaskel an elez hoc'h euz disprijet!

Setu aze ar c'homzou a garfemp hirio da gass beteg discouarn Kerneviz ha Tregeriz. Ar Juzevien a strake ho dent en ho fenn o clevet sant Stephan. Ne c' houlennomp ket muioc'h digant Kerneviz ha Tregeriz, nemed caout a raffe Doue din ac'hanomp euz an henor a reaz ar Juzevien da zant Stephan : d'hor laza a daoliou mein. An hanter vella euz al labour o deuz great. Pa girint e peurachuint anezhan, rag stanka hor ginou d'eomp, en dra vevimp, $n$ 'her graint biken. Torr he Benn.

(Au reste du département nous ne voyons pas de meilleur compliment à faire que celui que fit autrefois saint Étienne aux Juifs.

Têtes dures, oreilles de fonte, cœurs d'acier que vous êtes, dit-il, vous avez de tout temps été les ennemis de l'esprit divin; vous avez mis à mort l'Homme Juste; sa loi, qui vous était apportée par les ailes des anges, vous l'avez méprisée!

Voici les paroles que nous aimerions envoyer aujourd'hui aux oreilles des Cornouaillais et des Trégorois. Les Juifs avaient les dents qui crissaient dans leur bouche en entendant saint Étienne.

12. $\mathrm{F} \& \mathrm{~B} \mathrm{n}^{\circ} 36$ (7 octobre 1865$)$. 
Nous n'en attendons pas plus des Cornouaillais et des Trégorois, à moins que Dieu ne nous trouve dignes de l'honneur que firent les juifs à saint Étienne : de nous lapider. Ils ont déjà fait plus que la moitié du travail. Ils l'achèveront quand ils voudront, car nous clouer le bec, ils ne le feront jamais tant que nous vivrons. Torr-he Benn.)

On comprendra qu'un tel article n'a pas dû plaire à tous les lecteurs de Feiz ha Breiz et que certains collaborateurs de la revue aient pris leurs distances. Théophile de Pompéry qui avait écrit une série d'articles sur l'agriculture a dû apprécier modérément que Feiz ha Breiz appelle à voter contre lui parce que républicain.

Si Goulven Morvan ne faisait pas ouvertement de politique, ses successeurs remplissaient des pages relatant les débats à la Chambre. Le ton y est souvent vif et parfois injurieux : «arjuzeo teurs Naquet, ar born Gambetta... ». Les phrases sont souvent si longues et l'usage du point-virgule si courant que le lecteur peu habitué pourrait se perdre. On trouve là l'influence évidente de la solide culture latine de ces hommes d'Église. Un autre élément stable du style de Feiz ha Breiz est l'influence des Saintes Écritures. Des phrases entières des Pères de l'Église (Tertulien, Méliton de Sardes, Clément d'Alexandrie, etc.) sont ainsi traduites et recyclées dans Feiz ha Breiz. Méliton de Sardes est ainsi plagié dans un article narrant les agapes anthropophages d'un roi des îles Fidji ${ }^{13}$.

\section{Conclusion}

En conclusion, après dix ans de succès, Feiz ha Breiz prend un tour de plus en plus violemment monarchiste alors que la population et donc son lectorat se laissent gagner peu à peu par les idées républicaines. La fin était prévisible et même la plume de talent qu'était Gabriel Milin n'y pouvait rien. Même si la qualité littéraire de ses articles est grande, le lecteur devait tout de même se lasser de le voir faire feu de tout bois contre le gouvernement impie et les républicains. Devenu au fil des ans un outil de propagande monarchiste, Feiz ha Breiz n'a même pas survécu un an à la mort du comte de

13. JMN, «Sakobau, an debrer tud», Feiz ha Breiz n 10,6 mars 1880. 
Chambord (29 octobre 1883) qui laissait les légitimistes dans le plus grand désarroi.

Le bretonnant d'aujourd'hui constate que Feiz ha Breiz a joué un rôle très important dans l'histoire si houleuse de l'orthographe du breton. Il a aussi permis de confronter la réforme de Le Gonidec à la langue quotidienne du peuple. De cette confrontation rendue possible par la compétence et la personnalité d'hommes comme Goulven Morvan est née une synthèse dont les écrivains, journalistes, linguistes et enseignants bretonnants du XXI ${ }^{\mathrm{e}}$ siècle pourraient tirer grand profit. À un siècle et demi de distance et dans une situation sociolinguistique fort différente, son souci d'instruire et de distraire dans une langue élégante et vive nous offre un modèle : son art consommé du néologisme, ses explications limpides, son sens de l'humour et de la formule font de Goulven Morvan un auteur incontournable.

\section{Bibliographie}

BROUDIC Fañch, La pratique du breton de l'Ancien-Régime à nos jours, Rennes, PUR, 1995.

CHOPLIN Cédric, La représentation des peuples exotiques et des missions dans Feiz ha Breiz (1865-1884), Thèse de doctorat sous la direction de Gwendal Denis, Université Rennes 2, 2009.

RAOUL Lukian, Un siècle de journalisme breton, Le Guilvinec, Le Signor, 1981.

LAGRÉE Michel, Religion et cultures en Bretagne. 1850-1950, Paris, Fayard, 1992.

CALVEZ Ronan, «Un paysanisme breton» Feiz ha Breiz et la société bretonne (1865-1875), Brest, Mémoire de maîtrise, 1993.

LE Dû Jean, Le BerRe Yves, Textes choisis dans Feiz ha Breiz, Brest, Studi $n^{\circ}$ 11, avril 1979. 
